\title{
Comportamento agronômico de porta-enxertos de videira com resistência ao declínio de plantas jovens nas condições do estado de Santa Catarina
}

\author{
Marco Antonio Dalbó e Nelson Pires Feldberg ${ }^{2}$
}

\begin{abstract}
Resumo - O declínio de plantas jovens, causado pela ação conjunta da pérola-da-terra ou margarodes (Eurhizococcus brasiliensis) e fungos patogênicos (Cylindrocarpon, Phaeoacremonium e outros), é um problema grave em vinhedos do Sul do Brasil. Para diminuir as perdas provocadas por esse problema fitossanitário pode-se adotar o uso de porta-enxertos resistentes, mas seu uso não está difundido nessa região. Neste trabalho avaliou-se as características agronômicas do cultivar Moscato Embrapa enxertado nesses porta-enxertos em comparação com Paulsen 1103 e R99, utilizados como referência. De uma forma geral todos os porta-enxertos testados podem ser utilizados para produção de uva. Os porta-enxertos tropicais IAC 313 Tropical, IAC571-6 Jundiaí e IAC 766 Campinas induziram a brotação precoce comparada a Paulsen 1103, ao contrário de VR043-43 e Dog Ridge. Mesmo os porta-enxertos tropicais, sem dormência no inverno, comportam-se como porta-enxertos normais quando enxertados. O IAC 572 Jales tem a vantagem de não induzir a brotação precoce, mas provoca vigor excessivo da planta, exigindo práticas especiais de manejo para controlar o desenvolvimento vegetativo.
\end{abstract}

Termos para indexação: Vitis sp.; Cylindrocarpon sp.; pérola-da-terra; melhoramento genético.

\section{Agronomic behavior of grape rootstocks resistant to young vine decline in the conditions of the state of Santa Catarina Brazil}

\begin{abstract}
Young vine decline caused by the joint action of ground pearl or margarodes (Eurhizococcus brasiliensis) and pathogenic fungi (Cylindrocarpon, Phaeoacremonium and others) is a major problem in many vineyards of the South of Brazil. Resistant rootstocks can be used to reduce the losses caused by this phytosanitary problem; however, these rootstocks are not used on a large scale in the South of Brazil. This study evaluated the agronomic characteristics of the cultivar Moscato Embrapa grafted on these rootstocks, and compared to Paulsen 1103 and R99, which were used as reference. Tropical rootstocks IAC 313 Tropical, IAC571-6 Jundiaí and IAC 766 Campinas induced early bud break when compared to Paulsen 1103, unlike VR04343 and Dog Ridge. Even tropical rootstocks with no winter dormancy behave as normal rootstocks when grafted. IAC 572 Jales has the advantage of not inducing early bud break but causes excessive vigor, requiring special management practices to control vegetative development.
\end{abstract}

Index terms: Vitis sp.; Cylindrocarpon sp.; ground pearl; genetic improvement.

\section{Introdução}

Os porta-enxertos de videira foram desenvolvidos principalmente para resistência à filoxera, bem como para a adaptação a diferentes condições do solo (REISCH et al., 2012). No sul do Brasil, a ocorrência de declínio e morte de plantas jovens de videira levou à busca de porta-enxertos que possam resolver também esse problema, que é severo em alguns locais. No Vale do Rio do Peixe, a maior região vinícola de Santa Catarina, esse problema levou a uma redução significativa da área plantada nas décadas de 1980 e 90 (SCHUCK et al., 2001).

A principal causa atribuída a essa síndrome é a pérola-da-terra ou margarodes (Eurhizococcus brasiliensis). É uma cochonilha de raízes que ataca uma grande variedade de hospedeiros, mas é particularmente prejudicial à videira (BOTTON et al., 2000). No entanto, existem evidências de que os danos causados pela pérola-da-terra em raízes de videira levam à infecção por diversas espécies de fungos patogênicos do solo, como Cylindrocarpon, Phaeoa- cremonium e outros (GARRIDO et al., 2004). Os sintomas são semelhantes ao "declínio da videira jovem" e outras doenças da videira (doença de Petri, esca) causadas por este tipo de fungo e que foram relatados em outros países (GRAMAJE \& ARMENGOL, 2011; GATICA et al., 2001; STAMP, 2001). Esse problema fitossanitário pode ser manejado pelo uso de porta-enxertos com uma forma mais complexa de resistência, devido à multiplicidade de fatores envolvidos.

Ensaios de campo com porta-enxertos em áreas com alta incidência de declínio de videira mostraram que os por-

${ }^{2}$ Engenheiro-agrônomo, MSc., Embrapa Canoinhas, e-mail: nelson.feldberg@embrapa.br. 
ta-enxertos tradicionais são altamente suscetíveis (DALBÓ et al., 2016). Híbridos de espécies tropicais (Vitis caribaea, $V$. cinerea) apresentaram os maiores níveis de resistência, enquanto híbridos de Muscadinia rotundifolia (VR 043-43 e VR 039-16) e Dog Ridge (V. champinii) apresentaram resistência intermediária (DALBÓ et al., 2016). Híbridos de V. caribaea, como o IAC 572, foram criados para regiões tropicais, onde têm desempenho superior em termos de produtividade (TERRA et al., 2001; PAULETTO et al., 2001a). Em regiões mais frias, como no Meio-Oeste catarinense, características tropicais, como a ausência de dormência hibernal, são fatores limitantes ao seu uso, devido à indução de brotação precoce e aos danos causados por geadas em plantas jovens. Além disso, esses porta-enxertos induzem um vigor excessivo na copa (DALBÓ et al., 2011).

Considerando que os porta-enxertos que apresentaram maior resistência ao declínio da videira não são comumente utilizados na vitivinicultura do sul do Brasil, foi estudado o desempenho agronômico desses materiais em comparação com porta-enxertos tradicionais, visando avaliar o seu potencial em termos de produção e qualidade da uva nas condições do Meio-Oeste de Santa Catarina.

\section{Material e métodos}

O experimento foi instalado em uma propriedade privada no município de Videira, Santa Catarina (altitude: $855 \mathrm{~m}$, latitude: $26^{\circ} 56^{\prime} 09^{\prime \prime} \mathrm{S}$, longitude: $\left.51^{\circ} 14^{\prime} 41^{\prime \prime} W\right)$. O solo é classificado como Cambissolo Háplico Eutrófico (EMBRAPA, 2004).

Sete porta-enxertos foram testados, sendo dois deles considerados tradicionais (Paulsen 1103 e R99) e os demais escolhidos com base em estudos anteriores que indicaram serem menos suscetíveis ao declínio de videira. Foram avaliados os seguintes porta-enxertos, com sua origem (cruzamento): Paulsen 1103 (V. berlandieri x V. rupestris); R99 (V. berlandieri $\times$ V. rupestris); VR 043-43 (V. vinifera x Muscadinia rotundifolia);
Dog Ridge (V. champinii); IAC 313 Tropical ( $V$. cinerea $\times$ Golia); IAC 572 Jales (V. caribaea $\times$ 101-14); IAC 571-6 Jundiaí (V. vinifera $\times$ V. caribaea); IAC 766 Campinas (V. caribaea $\times 106-8$ ). As parcelas consistiram em quatro plantas espaçadas de $3,5 \times 1,5 \mathrm{~m}$.

Os porta-enxertos foram plantados no local definitivo em agosto de 2009, sendo enxertados com o cultivar Moscato Embrapa em agosto de 2010. No ano seguinte, alguns enxertos que faIharam no ano anterior foram refeitos. O manejo do solo consistiu no controle da vegetação com herbicidas ao longo das linhas e roçada entre linhas. O controle de doenças foi feito através de pulverizações periódicas com fungicidas, conforme recomendado para a cultura (EPAGRI, 2005).

Para avaliação da produtividade e qualidade dos frutos, os rendimentos iniciais foram descartados e considerados apenas os dados obtidos nas safras de 2014 a 2017 (4 ao $7^{\circ}$ ano após a enxertia). As avaliações de qualidade da uva foram baseadas em amostras de 100 bagas coletadas aleatoriamente na parcela. O mosto foi analisado quanto a sólidos solúveis ( ${ }^{\circ} \mathrm{Brix}$ ) em refratômetro de bancada (Nova Instruments), pH (phmetro Diagtech) e acidez total titulável (meq ${ }^{-1}$ ). A avaliação dos dados fenológicos (início de brotação) foi estendida de 2012 a 2015. Considerou-se que as plantas estavam começando a brotar quando a maioria das gemas terminais atingiu o estágio de ponta verde.

O delineamento experimental foi em blocos ao acaso, com cinco repetições. A análise estatística (Anova) foi feita utilizando o software $\mathrm{R}$ (versão 3.4.2). O teste de comparação de médias (Tukey) é apresentado para a média dos anos de avaliação no caso de variáveis em que não houve interação significativa entre ano e porta-enxerto. Por outro lado, no caso de interação significativa das variáveis os resultados foram apresentados em cada período experimental.

\section{Resultados e discussão}

As observações fenológicas indicam que o porta-enxerto tem influência sobre o início de brotação do cultivar copa (Moscato Embrapa) e que essas diferenças são constantes na maioria dos anos (Figura 1). Considerando-se o portaenxerto Paulsen 1103 como referência, observou-se que os porta-enxertos VR 043-43, Dog Ridge e R99, induziram uma brotação posterior. Por outro lado, os porta-enxertos IAC 313 Tropical, IAC 571-6 Jundiaí e IAC 766 Campinas tenderam a antecipar a brotação. Esses três porta-enxertos são híbridos de espécies tropicais, nas quais não há um período

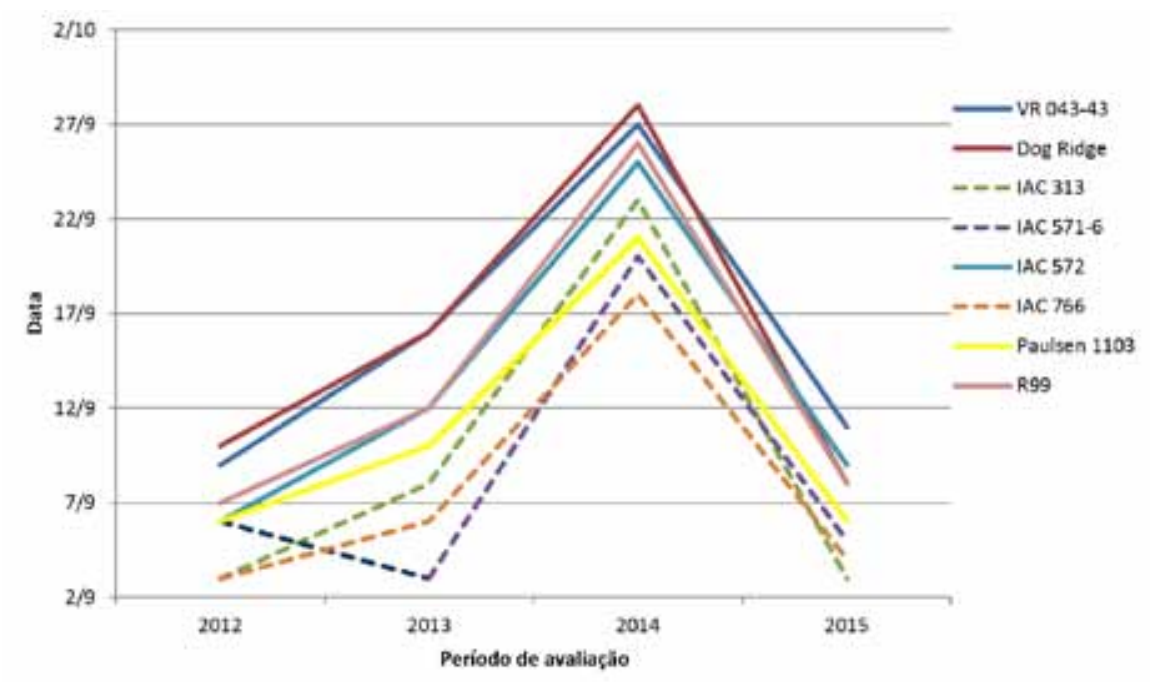

Figura 1. Data de início de brotação do cultivar 'Moscato Embrapa' enxertado sobre diferentes porta-enxertos no período de 2012 a 2015 em Videira, SC, Brasil

Figure 1. Bud break date of the cultivar 'Moscato Embrapa' grafted onto different rootstocks in the 2012 to 2015 period in Videira, SC, Brazil 
de dormência definido, o que poderia levar a uma antecipação do início da brotação. No entanto, o IAC 572 Jales que também apresenta características tropicais, teve a brotação da copa de média a tardia, geralmente alguns dias depois de Paulsen 1103.

A brotação tardia é particularmente vantajosa em anos com ocorrência de geadas tardias, como ocorreu no período de 2015. Nesse ano, as condições climáticas foram bastante atípicas, com um inverno ameno, mas com geadas tardias (10 e 11 de setembro) quando todas as plantas já tinham começado a brotar. Embora tenha havido dano em todas as combinações de copa e porta-enxerto, as perdas de produção na safra seguinte (2016) foram, em geral, maiores no caso dos porta-enxertos que anteciparam o início da brotação (Tabela 1). Nos outros anos, quando as condições climáticas foram normais, as diferenças de produtividade entre os porta-enxertos foram menores. Mesmo assim, não houve interação significativa entre porta-enxerto e safra. Na média geral, as maiores produtividades foram proporcionadas pelos porta-enxertos VR 043-43, IAC 572 Jales, Paulsen 1103 e IAC 571-6 Jundiaí, que não diferiram entre si. Os porta-enxertos R99, IAC 313 Tropical e Dog Ridge foram os que resultaram em menor produtividade. No caso do R99, possivelmente está associado ao menor vigor das plantas (Tabela 2).

O IAC 572 Jales foi o porta-enxerto que induziu o maior vigor à copa e o R99, o de menor vigor. Os outros mostraram resultados intermediários (Tabela 2). O alto vigor da copa induzido pelos porta-enxertos da série IAC, particularmente pelo IAC 572 Jales, já havia sido observado em outros estudos (DALBÓ et al., 2011). No presente experimento foram necessárias três intervenções de poda verde para controle do crescimento vegetativo, principalmente no caso dos porta-enxertos mais vigorosos.

O crescimento excessivo de plantas induzido pelo IAC 572 Jales parece ser o principal fator negativo deste portaenxerto. A tendência de ocorrer aborto

Tabela 1. Produtividade do cultivar 'Moscato Embrapa' enxertado sobre diferentes portaenxertos no período de 2012 a 2015 em Videira, SC, Brasil

Table 1. Productivity of 'Moscato Embrapa' cultivar grafted on different rootstocks from 2012 to 2015 in Videira, SC, Brazil

\begin{tabular}{llllll}
\hline Porta-enxerto & $\mathbf{2 0 1 4}$ & $\mathbf{2 0 1 5}$ & $\mathbf{2 0 1 6}$ & $\mathbf{2 0 1 7}$ & Média \\
\hline & 25,3 & 25,8 & 14,2 & 31,9 & 24,3 a* \\
VR 043-43 & 17,1 & 19,0 & 8,8 & 21,2 & $16,5 \mathrm{c}$ \\
Dog Ridge & 14,0 & 17,5 & 6,2 & 21,6 & $14,8 \mathrm{c}$ \\
IAC 313 Tropical & 22,2 & 20,6 & 15,5 & 22,9 & $20,3 \mathrm{abc}$ \\
IAC 571-6 Jundiaí & 23,4 & 22,3 & 21,1 & 26,1 & $23,2 \mathrm{a}$ \\
IAC 572 Jales & 20,8 & 16,5 & 8,3 & 20,9 & $16,6 \mathrm{bc}$ \\
IAC 766 Campinas & 25,3 & 24,5 & 9,7 & 29,1 & $22,2 \mathrm{ab}$ \\
\hline Paulsen 1103 & 16,6 & 15,2 & 6,6 & 21,3 & $14,9 \mathrm{c}$ \\
\hline R99 & 26,1 & 33,4 & 46,9 & 23,3 & 30,2 \\
\hline CV(\%) & & & & \\
\hline
\end{tabular}

*Significativo a $5 \%$ pelo teste de Tukey

Tabela 2. Vigor das plantas estimado pelo material retirado na poda de inverno do cultivar 'Moscato Embrapa' enxertado sobre oito porta-enxertos, no período de 2012 a 2015 em Videira, SC, Brasil

Table 2. Plant vigor, estimated by the material removed from the winter pruning, of 'Moscato Embrapa' cultivar grafted on eight rootstocks, in the period from 2012 to 2015 in Videira, SC, Brazil

\begin{tabular}{lccccc}
\hline Porta-enxerto & $\mathbf{2 0 1 4}$ & $\mathbf{2 0 1 5}$ & $\mathbf{2 0 1 6}$ & $\mathbf{2 0 1 7}$ & Média \\
\hline & \multicolumn{5}{c}{$\mathrm{Kg} \mathrm{pl}^{-1}$} \\
VR 043-43 & $2,26 \mathrm{~b} *$ & $1,38 \mathrm{~cd}$ & $1,36 \mathrm{bc}$ & $1,06 \mathrm{~d}$ & 1,51 \\
Dog Ridge & $2,08 \mathrm{~b}$ & $1,49 \mathrm{bcd}$ & $1,15 \mathrm{bc}$ & $1,58 \mathrm{bc}$ & 1,57 \\
IAC 313 Tropical & $2,59 \mathrm{~b}$ & $1,88 \mathrm{bc}$ & $1,84 \mathrm{ab}$ & $1,57 \mathrm{bc}$ & 1,97 \\
IAC 571-6 Jundiaí & $2,96 \mathrm{~b}$ & $2,11 \mathrm{~b}$ & $1,50 \mathrm{~b}$ & $1,65 \mathrm{~b}$ & 2,05 \\
IAC 572 Jales & $4,23 \mathrm{a}$ & $3,08 \mathrm{a}$ & $2,38 \mathrm{a}$ & $2,45 \mathrm{a}$ & 3,03 \\
IAC 766 Campinas & $2,44 \mathrm{~b}$ & $2,09 \mathrm{~b}$ & $1,28 \mathrm{bc}$ & $1,22 \mathrm{~cd}$ & 1,76 \\
Paulsen 1103 & $2,30 \mathrm{~b}$ & $1,57 \mathrm{bcd}$ & $1,28 \mathrm{bc}$ & $1,33 \mathrm{bcd}$ & 1,62 \\
R99 & $2,11 \mathrm{~b}$ & $0,95 \mathrm{~d}$ & $0,69 \mathrm{c}$ & $1,10 \mathrm{~d}$ & 1,21 \\
\hline CV(\%) & 21,66 & 18,59 & 25,13 & 13,74 & 21,25 \\
\hline
\end{tabular}

*Significativo a $5 \%$ pelo teste de Tukey

floral foi observada eventualmente em algumas plantas com este porta-enxerto, mas não chegou a comprometer a produção.

Os porta-enxertos vigorosos tendem a prolongar a maturação dos frutos em comparação com genótipos de baixo vigor (POUGET, 1986). Além disso, se um porta-enxerto diminui o vigor da variedade copa, há uma diminuição do adensamento do dossel vegetativo, fazendo com que os cachos fiquem mais expostos à radiação solar (SPAYD et al., 2002). Alguns estudos mostraram uma diminuição dos teores de sólido solúveis com o uso de porta-enxertos vigorosos, como os da série IAC, em comparação com outros de menor vigor para os cultivares copa Niágara Rosada (PAULETTO et al., 2001b) e Bordô (MOTA et al., 2009). Entretanto, em outro estudo não foram encontradas diferenças significativas com relação a essas características para Isabel, no norte do Paraná (SATO et al., 2009), indicando que isso pode variar conforme as condições experimentais.

No presente caso, com base nas variáveis de avaliação da qualidade dos frutos não foram observadas diferenças consistentes entre os porta-enxertos ao longo dos anos. O R99, de baixo vigor, não esteve entre os porta-enxertos que forneceram maior conteúdo de sólidos 
solúveis (Tabela 3), mas foi o que resultou no menor nível de acidez (Tabela 4). O IAC 572 Jales resultou em menor teor de sólidos solúveis (Tabela 3). No entanto, combinações de copa/porta-enxerto altamente vigorosas frequentemente resultam em ciclos vegetativos mais longos e demoram mais para atingir a maturidade. Nesse experimento, a data de colheita foi a mesma para todos os porta-enxertos, o que desfavoreceu a completa maturação da uva em plantas enxertadas no IAC 572 Jales. O mesmo se verifica com relação aos dados de $\mathrm{pH}$ do mosto (Tabela 5). Ocorreram diferenças significativas entre porta-enxertos nos anos 2016 e 2017, porém as diferenças não foram consistentes no decorrer dos anos. Assim, não é possível destacar um porta-enxerto que induza melhores características qualitativas na produção do cultivar copa.

A área do experimento havia sido escolhida pelo fato de que não haviam sido observados problemas claros de declínio e morte de plantas na propriedade. No período em que o experimento foi conduzido, também não foram observados sintomas nas plantas avaliadas. Desse modo, pode-se considerar que os resultados refletem basicamente o efeito das características agronômicas dos porta-enxertos sobre a copa avaliada.

De acordo com as observações e os resultados obtidos até o momento, é possível utilizar porta-enxertos com características tropicais, como a série IAC, em regiões frias do sul do Brasil. O IAC 572 Jales se destaca por não antecipar a brotação do cultivar copa em comparação com o porta-enxerto de referência para esta região (Paulsen 1103) e por ser um dos porta-enxertos que induziram maior produtividade ao longo dos anos, juntamente com o VR 043-43 e o Paulsen 1103. O IAC 572 Jales também foi um dos mais resistentes ao declínio e morte de videiras jovens em trabaIhos anteriores (DALBÓ et al., 2016; LOURENÇÃO et al., 2002). No entanto, sua principal desvantagem é o alto vigor que imprime à copa, o que requer práticas de manejo diferenciadas para
Tabela 3. Teor de sólidos solúveis ('Brix) do mosto de uva do cultivar 'Moscato Embrapa' enxertado sobre oito porta-enxertos no período de 2012 a 2015 em Videira, SC, Brasil Table 3. Soluble solids content ( ${ }^{\circ} \mathrm{Brix}$ ) of Moscato Embrapa grape must grafted on eight rootstocks from 2012 to 2015 in Videira, SC, Brazil

\begin{tabular}{llllll}
\hline Porta-enxerto & $\mathbf{2 0 1 4}$ & $\mathbf{2 0 1 5}$ & $\mathbf{2 0 1 6}$ & $\mathbf{2 0 1 7}$ & Média \\
\hline VR 043-43 & $19,0 \mathrm{ab}$ & $16,2 \mathrm{a}$ & $18,3 \mathrm{ab}$ & $16,5 \mathrm{a}$ & 17,5 \\
Dog Ridge & $18,8 \mathrm{ab}$ & $16,7 \mathrm{a}$ & $17,9 \mathrm{abc}$ & $17,8 \mathrm{a}$ & 17,8 \\
IAC 313 Tropical & $18,9 \mathrm{ab}$ & $16,2 \mathrm{a}$ & $18,3 \mathrm{a}$ & $17,2 \mathrm{a}$ & 17,7 \\
IAC 571-6 Jundiaí & $19,2 \mathrm{ab}$ & $16,6 \mathrm{a}$ & $17,3 \mathrm{c}$ & $16,8 \mathrm{a}$ & 17,5 \\
IAC 572 Jales & $18,4 \mathrm{~b}$ & $15,5 \mathrm{a}$ & $17,3 \mathrm{bc}$ & $17,2 \mathrm{a}$ & 17,1 \\
IAC 766 Campinas & $19,8 \mathrm{a}$ & $16,8 \mathrm{a}$ & $18,2 \mathrm{abc}$ & $17,4 \mathrm{a}$ & 18,1 \\
Paulsen 1103 & $18,7 \mathrm{ab}$ & $16,3 \mathrm{a}$ & $18,4 \mathrm{a}$ & $16,5 \mathrm{a}$ & 17,5 \\
R99 & $18,3 \mathrm{~b}$ & $16,7 \mathrm{a}$ & $17,8 \mathrm{abc}$ & $17,0 \mathrm{a}$ & 17,5 \\
\hline CV(\%) & 4,0 & 5,4 & 2,7 & 4,6 & 4,0 \\
\hline
\end{tabular}

*Significativo a $5 \%$ pelo teste de Tukey

Tabela 4. Acidez total do mosto de uva do cultivar 'Moscato Embrapa' enxertado sobre oito porta-enxertos, no período de 2012 a 2015 em Videira, SC, Brasil

Table 4. Total acidity of grape must cultivar 'Moscato Embrapa' grafted on eight rootstocks, in the period from 2012 to 2015 in Videira, SC, Brazil

\begin{tabular}{lcllll}
\hline Porta-enxerto & $\mathbf{2 0 1 4}$ & $\mathbf{2 0 1 5}$ & $\mathbf{2 0 1 6}$ & $\mathbf{2 0 1 7}$ & Média \\
\hline & \multicolumn{5}{c}{ meq L-1 $^{-}$} \\
\cline { 2 - 6 } VR 043-43 & 70,3 & 76,9 & 84,8 & 103,4 & $83,9 \mathrm{ab} *$ \\
Dog Ridge & 65,6 & 78,8 & 83,2 & 106,0 & $83,4 \mathrm{ab}$ \\
IAC 313 Tropical & 65,8 & 74,8 & 84,7 & 93,8 & $79,7 \mathrm{~b}$ \\
IAC 571-6 Jundiaí & 60,4 & 77,6 & 82,4 & 98,6 & $79,8 \mathrm{~b}$ \\
IAC 572 Jales & 71,3 & 78,2 & 93,9 & 109,9 & $88,3 \mathrm{ab}$ \\
IAC 766 Campinas & 64,6 & 81,2 & 87,2 & 100,7 & $83,4 \mathrm{ab}$ \\
Paulsen 1103 & 63,5 & 73,4 & 89,9 & 101,5 & $82,1 \mathrm{~b}$ \\
\hline R99 & 52,0 & 66,9 & 74,8 & 94,1 & $72,0 \mathrm{c}$ \\
\hline CV(\%) & 9,7 & 8,4 & 5,7 & 5,3 & 7,0 \\
\hline
\end{tabular}

*Significativo a $5 \%$ pelo teste de Tukey

Tabela 5. pH do mosto de uva do cultivar 'Moscato Embrapa' enxertado sobre oito portaenxertos no período de 2012 a 2015 em Videira, SC, Brasil

Table 5. $\mathrm{pH}$ of grape must of 'Moscato Embrapa' cultivar grafted on eight rootstocks from 2012 to 2015 in Videira, SC, Brazil

\begin{tabular}{llllll}
\hline Porta-enxerto & 2014 & 2015 & 2016 & 2017 & Média \\
\hline VR 043-43 & $3,19 \mathrm{a}$ & $3,15 \mathrm{a}$ & $3,43 \mathrm{a}$ & $3,42 \mathrm{bc}$ & 3,28 \\
Dog Ridge & $3,17 \mathrm{a}$ & $3,14 \mathrm{a}$ & $3,40 \mathrm{ab}$ & $3,36 \mathrm{abc}$ & 3,27 \\
IAC 313 Tropical & $3,19 \mathrm{a}$ & $3,12 \mathrm{a}$ & $3,39 \mathrm{ab}$ & $3,38 \mathrm{ab}$ & 3,27 \\
IAC 571-6 Jundiaí & $3,24 \mathrm{a}$ & $3,19 \mathrm{a}$ & $3,34 \mathrm{ab}$ & $3,35 \mathrm{abc}$ & 3,28 \\
IAC 572 Jales & $3,21 \mathrm{a}$ & $3,17 \mathrm{a}$ & $3,36 \mathrm{ab}$ & $3,32 \mathrm{c}$ & 3,26 \\
IAC 766 Campinas & $3,26 \mathrm{a}$ & $3,17 \mathrm{a}$ & $3,39 \mathrm{ab}$ & $3,41 \mathrm{a}$ & 3,30 \\
Paulsen 1103 & $3,22 \mathrm{a}$ & $3,14 \mathrm{a}$ & $3,31 \mathrm{~b}$ & $3,38 \mathrm{abc}$ & 3,26 \\
R99 & $3,22 \mathrm{a}$ & $3,22 \mathrm{a}$ & $3,40 \mathrm{ab}$ & $3,34 \mathrm{bc}$ & 3,30 \\
\hline CV(\%) & 1,60 & 1,57 & 1,02 & 0,90 & 1,30 \\
\hline *Significativo a 5\% pelo teste de Tukey & & & &
\end{tabular}


reduzir o crescimento vegetativo, como podas verdes intensivas, espaçamentos maiores entre plantas e redução do uso de fertilizantes ao longo do ciclo. Pode ser indicado para áreas de replantio, principalmente para cultivares de uva de mesa ou para produção de suco de uva, onde o vigor elevado é mais tolerado. Para a produção de vinho, entretanto, o vigor excessivo é um defeito difícil de ser contornado com práticas culturais. Isso somente poderá ser obtido com o desenvolvimento de novos porta-enxertos que sejam ao mesmo tempo resistentes e menos vigorosos.

\section{Conclusão}

1. É possível utilizar porta-enxertos com características tropicais, como a série IAC, em regiões frias do sul do Brasil;

2. Com exceção do IAC 572 Jales, os porta-enxertos da série IAC induziram uma brotação mais precoce do cultivar Moscato Embrapa em relação ao porta-enxerto Paulsen 1103;

3. As maiores produtividades foram obtidas com os porta-enxertos VR 043-43, IAC 572 Jales e Paulsen 1103;

4. O maior vigor de plantas foi obtido com os porta-enxerto IAC 572 Jales, seguido do IAC 571-6 Campinas.

\section{Agradecimentos}

Ao Conselho Nacional de Desenvolvimento Científico e Tecnológico (CNPq) pelo financiamento e à dra. Simone $\mathrm{S}$. Werner pelo auxílio na realização de análises estatísticas.

\section{Referências}

BOTTON, M.; HICKEL, E.R.; SORIA, S.J.; TEI$X E I R A, I$. Bioecologia e controle da pérolada-terra Eurhizococcus brasiliensis (Hempel, 1922) (Hemiptera:Margarodidae) na cultura da videira. Bento Gonçalves: Embrapa Uva e Vinho, 2000. 23p. (Circular Técnica, 27).

DALBÓ, M.A.; SCHUCK, E.; BASSO, C. Influence of rootstock on nutrient content in grape petioles. Revista Brasileira de Fruticultura, Jaboticabal, v. 33, n. 3, p. 941-947, 2011.

DALBÓ, M.A.; ARIOLI, C.J.; SILVA, M.L. Resistance of rootstocks to grapevine decline and dieback in southern Brazil. Acta Horticulturae, Leuven, n. 1127, p. 65-70, 2016.

EMBRAPA. Solos do Estado de Santa Catarina. Rio de Janeiro: Embrapa Solos; CNPS, 2004. 745p. (Boletim de desenvolvimento e pesquisa).

EPAGRI. Normas técnicas para o cultivo da videira em Santa Catarina. Florianópolis: Epagri, 2005. 67p. (Sistemas de Produção, 33).

GARRIDO, L.R.; SONEGO, O.R.; URBEN, A.F. Cylindrocarpon destructans, causador do "pé-preto" da videira no Rio Grande do Sul. Fitopatologia Brasileira, Brasília, DF, v. 29, n. 5, p. 548-550, 2004.

GATICA, M.; CÉSARI, C.; MAGNIN, S.; DUPONT, J. Phaeoacremonium species and Phaeomoniella chlamydospora in vines showing "hoja de malvón" and Young vine decline symptoms in Argentina. Phytopath Mediterranea, Firenze, v. 40, p. 317-324, 2001.

GRAMAJE, D.; ARMENGOL, J. Fungal trunk pathogens in the grapevine propagation process: potential inoculum sources, detection, identification, and management strategies. Plant Disease, [S.I.], v.95, p.1040-1055, 2011.

LOURENÇÃO, A.L.; TERRA, M.M.; PIRES, E.J.P.; POMMER, C.V.; AMBROSANO, G.M.V. Comportamento de porta-enxertos de videira em solo infestado pela pérola-da-terra. Revista de Agricultura, Piracicaba, v.77, n. 1, p.57-64, 2002.

MOTA, R.V.; SOUZA, C.R.; FAVERO, A.C.; SILVA, C.P.C.; CARMO, E.L.; FONSECA, A.R.; REGINA, M.A. Produtividade e composição físico-química de bagas de cultivares de uva em distintos porta-enxertos. Pesquisa Agropecuária Brasileira, Brasília, DF, v. 44, n.6, p. 576-582, 2009.

PAULETTO, D.; MOURÃO FILHO, F. A. A.; KLUGE, R.A.; SCARPARE FILHO, R.A. Produção e vigor da videira "Niágara Rosada" relaciona- dos com o porta-enxerto. Pesquisa Agropecuária Brasileira, Brasília, DF, v. 36, n. 1, p. 115-121, 2001a.

PAULETTO, D.; MOURÃO FILHO, F.A.A.; KLUGE, R.A.; SCARPARE FILHO, R.A. Efeito do porta-enxerto na qualidade do cacho da videira "Niágara Rosada". Pesquisa Agropecuária Brasileira, Brasília, DF, v. 36, n. 7, p. 935-939, 2001b.

POUGET, R. Usefulness of rootstocks for controlling vine vigour and improving wine quality. In: KLIEWER, W.M. (Ed.). Symposium on grapevine canopy and vigor management, XXII IHC. Davis: Acta Horticulturae,1986. p.109-118.

REISCH, B.I.; OWENS, C.L.; COUSINS, P.S. Grape. In: BADENES, M.L.; BYRNE, D.H. (Eds.). Fruit Breeding. Springer: New York, 2012. p. 225-262.

SATO, A.J.; JUBILEU, B.; BERTOLUCCI, R.; CARIELO, M.; GUIRAUD, M.C.; FONSECA, I.C.B.; ROBERTO, S. Ripening evolution and physico-chemical characteristics of 'Isabel' grape on differents rootstocks in North of Parana. Semina: Ciências Agrárias, Londrina, v. 30, n. 1, p. 11-20, 2009.

SCHUCK, E.; DALBÓ, M.A.; ROSIER, J.P.; DUCROQUET, J.P. Porta-enxertos para a cultura da Videira. In: ENFRUTE, 4., 2001, Fraiburgo. Anais [...]. Caçador: Epagri, 2001. p.122-132.

STAMP, J.A. The contribution of imperfections in nursery stock to the decline of young vines in California. Phytopathologia Mediterranea, Firenze, v. 40, p.369-375, 2001.

SPAYD, S.E; TARARA, J.M.; MEE, D.L.; FERGUNSON, J.C. Separation of sunlight and temperature effects on the composition of Vitis vinifera cv. Merlot berries. American Journal of Enology and Viticulture, [S.I.], v.53, n. 3, p. 171-182, 2002.

TERRA, M.M.; POMMER, C.V.; PIRES, E.J.P.; RIBEIRO, I.J.A.; GALLO, P.B.; PASSOS, I.R.S. Produtividade de cultivares de uva para suco sobre diferentes porta-enxertos IAC, em Mococa, SP. Revista Brasileira de Fruticultura, Jaboticabal, v.23, n.2, p.382-386, 2001. 